\title{
Anti-SARS-CoV-2 IgY Isolated from Egg Yolks of Hens Immunized with Inactivated SARS-CoV-2 for Immunoprophylaxis of COVID-19
}

\author{
Haiyan Shen ${ }^{1} \cdot$ Yanxing $\mathrm{Cai}^{2} \cdot$ Huan $\mathrm{Zhang}^{3} \cdot$ Jie $\mathrm{Wu}^{3} \cdot \mathrm{Lin} \mathrm{Ye}^{4} \cdot$ Penghui Yang ${ }^{5} \cdot \mathrm{Xiaojun} \mathrm{Lin}^{4}(1) \cdot$ \\ Shibo Jiang ${ }^{2}$ (D) $\cdot$ Ming Liao ${ }^{1}$
}

Received: 29 November 2020 / Accepted: 8 February 2021 / Published online: 21 May 2021

(C) Wuhan Institute of Virology, CAS 2021

\section{Dear Editor,}

In the absence of vaccine, the mounting second wave of COVID-19 infections worldwide calls for the rapid development of human neutralizing antibodies to prevent and treat COVID-19 (Lee et al. 2020). Considering the high cost and long development cycle of human neutralizing antibodies, we turned to anti-SARS-CoV-2 IgY for cheap and fast scale up.

In particular, egg yolk antibody IgY, the evolutionary precursor of $\mathrm{IgG}$, is widely present in birds, reptiles, and amphibians, and it can be easily obtained on a large scale from egg yolk (Pereira et al. 2019). As a component of chicken eggs consumed by humans, IgY is expected to be safe and has been widely applied as prophylactics against respiratory infections because of its high binding affinity to antigens, thermostability, high resistance to $\mathrm{pH}$ variation,

Haiyan Shen, Yanxing Cai, and Huan Zhang contributed equally to this work.

\section{Supplementary Information The online version contains} supplementary material available at https://doi.org/10.1007/s12250021-00371-1.

Ming Liao

mliao@scau.edu.cn

$\triangle$ Shibo Jiang

shibojiang@fudan.edu.cn

$\triangle$ Xiaojun Lin

davidlin81@hotmail.com

1 Institute of Animal Health, Guangdong Academy of Agricultural Sciences, Guangzhou 510640, China

2 Key Laboratory of Medical Molecular Virology (MOE/NHC/ CAMS), School of Basic Medical Sciences, Fudan University, Shanghai 200032, China

3 Guangdong Provincial Center for Disease Control and Prevention, Guangzhou 511430, China

4 Chengdu YuKang Biotechnology Co., LTD, Chengdu 611633, China and cost-effectiveness (Abbas et al. 2019). Because of the advantages of IgY, we aimed to isolate anti-SARS-CoV-2 IgY from the yolks of eggs delivered by hens immunized with inactivated SARS-CoV-2 and evaluate its inhibitory activity against SARS-CoV-2 infection in vitro, with a hope to develop IgY-based immunoprophylactic or therapeutic for prevention or treatment of COVID-19.

Specifically, SARS-CoV-2 (20SF014-SARS-CoV-2) was expanded in Vero-E6 cells, collected, and stored at $-80{ }^{\circ} \mathrm{C}$ until use. Lohmann pink-shell laying hens were subcutaneously immunized with formaldehyde-inactivated SARS-CoV-2 and Freund's complete adjuvant, boosted twice at 2-3 week interval with the mixture of the inactivated virus and Freund's incomplete adjuvant on both wings $(0.5 \mathrm{~mL} / \mathrm{hen})$. One week after the final immunization, the eggs were collected, and crude $\operatorname{IgY}$ antibodies were extracted from the egg yolks using the water-soluble fraction method (Supplementary Materials). Purified IgY antibodies were confirmed by Western blotting (Fig. 1A). We then tested the neutralizing activity of these $\operatorname{IgY}$ antibodies against pseudotyped and live SARS-CoV-2 infection in vitro as previously described (Xia et al. 2020). As shown in Fig. 1B, 1C, the IgY antibodies were effective in neutralizing infection by both pseudotyped and live SARS$\mathrm{CoV}-2$ in a dose-dependent manner with half maximal inhibitory concentration $\left(\mathrm{IC}_{50}\right)$ of 9.26 and $44.31 \mu \mathrm{g} / \mathrm{mL}$, respectively, while the isotype control $\mathrm{IgY}$ antibodies had no inhibitory effect on pseudotyped and live SARS-CoV-2 infection at a concentration as high as $100 \mu \mathrm{g} / \mathrm{mL}$.

Next, we assessed the inhibitory activity of purified IgY antibodies on SARS-CoV-2 spike (S) protein-mediated cell-cell fusion as previously described (Xia et al. 2020). As shown in Fig. 1D, these $\operatorname{IgY}$ antibodies were also effective in inhibiting SARS-CoV-2 S-mediated cell-cell fusion in a dose-dependent manner with $\mathrm{IC}_{50}$ of $15.83 \mu \mathrm{g} / \mathrm{mL}$, while, again, no inhibitory activity was observed in the

5 Fifth Medical Center of Chinese, PLA General Hospital, Beijing 100039, China 
A

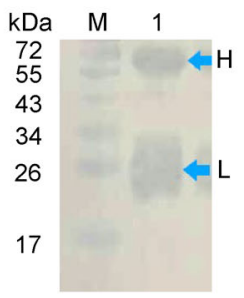

D

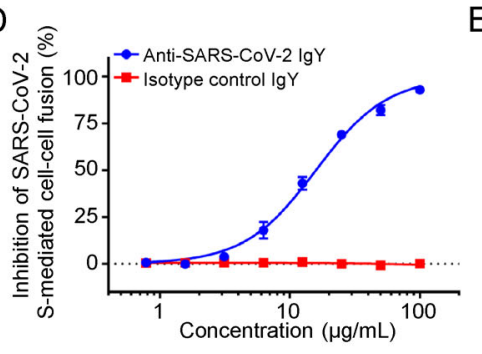

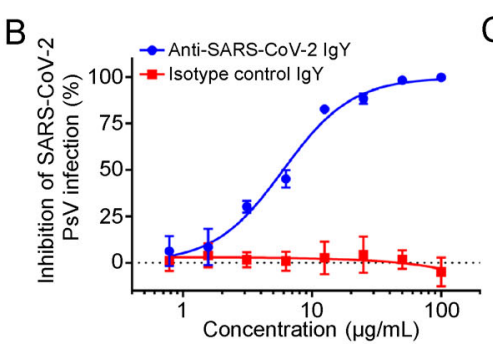

E

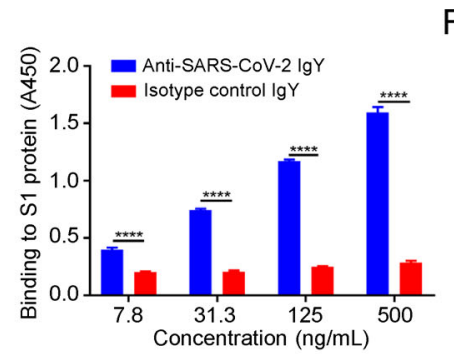

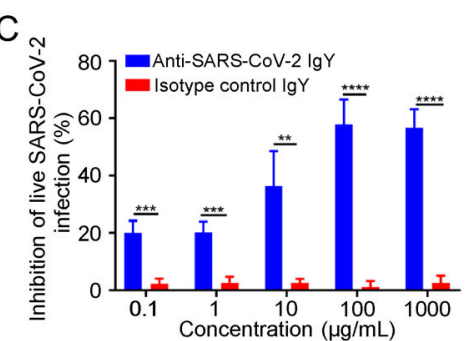

$\mathrm{F}$

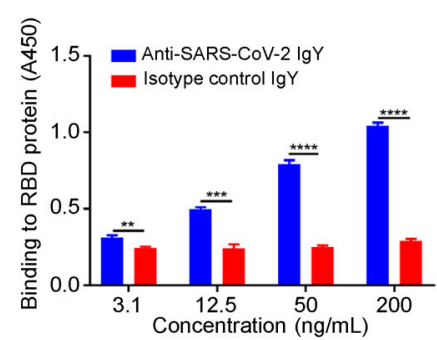

G

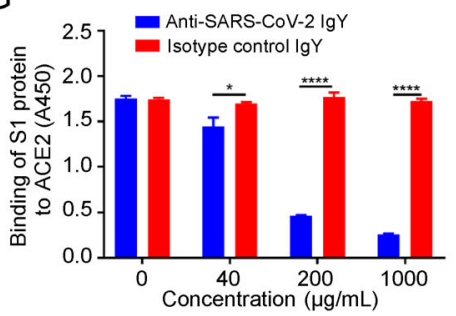

I

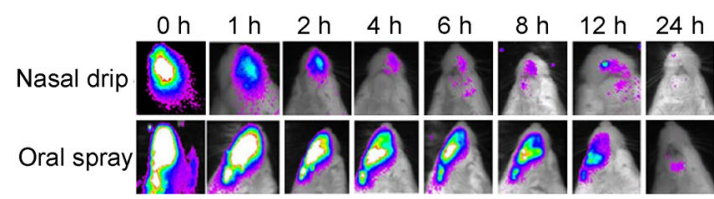

$\mathrm{H}$

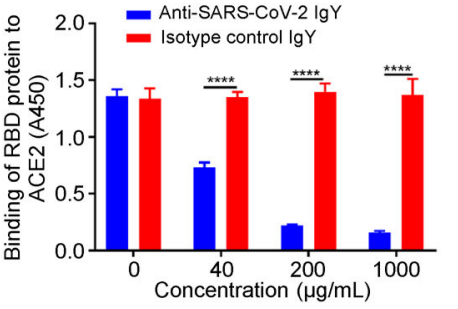

$J$

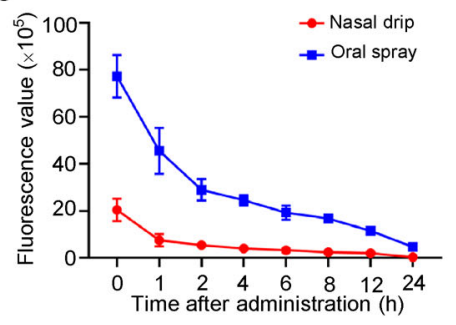

H Effect of anti-SARS-CoV-2 IgY antibodies on the binding of S1 protein $(\mathbf{G})$ and RBD $(\mathbf{H})$ to ACE2 was measured by ELISA. I, J Detection of anti-SARS-CoV-2 IgY in the nasal and oral cavities of mice was measured by in vivo imaging. Growth curves were obtained from serial measurements in three experiments (four or five animals per group). Experiments were repeated twice, and the data are presented as means \pm SD. GraphPad Prism 5.0 was used to perform statistical analysis, and a $P$ value of less than 0.05 was considered to indicate a significant difference, $* P<0.05 ; \quad * * P<0.01$; $* * * P<0.001 ; * * * * P<0.0001$.

epitopes and neutralizing antibodies from SARS-CoV- and SARS-CoV-2-infected patients, as well as hosts immunized with the inactivated vaccines that mainly target RBD (He et al. 2005, 2006; Du et al. 2009; Su et al. 2020). To illustrate the antiviral mechanism of anti-SARS-CoV-2 IgY antibodies, we performed an enzyme-linked immunosorbent assay (ELISA) to test whether these antibodies interact with S1 and RBD in S protein of SARS-CoV-2 and SARS-CoV-2 $S$ protein contains the major neutralizing 
whether they can block the binding of $\mathrm{S} 1$ and RBD to human ACE2. As shown in Fig. 1E, 1F, anti-SARS-CoV-2 $\mathrm{IgY}$ antibodies bound to $\mathrm{S} 1$ protein and RBD in a dosedependent manner, while isotype control IgY antibodies could not interact with either S1 or RBD. Notably, antiSARS-CoV-2 IgY antibodies could significantly block the binding of S1 or RBD to ACE2 in a dose-dependent manner, while the isotype control IgY antibodies could not inhibit interaction between $\mathrm{S} 1$ or RBD and ACE2 (Fig. 1G, $1 \mathrm{H})$. These results indicate that anti-SARS-CoV-2 IgY antibodies can bind to RBD in S1, thus blocking binding between RBD and ACE2 and inhibiting SARS-CoV-2 entry, fusion, and infection.

Ciliated nasal epithelial cells are particularly susceptible to SARS-CoV-2 infection owing to the high expression of ACE2, making the upper airways a key target for SARSCoV-2 infection (Hou et al. 2020). Previous studies have shown that IgY passive immunization can be used to prevent and treat virus infection in the respiratory tract (Abbas et al. 2019). Therefore, to test whether anti-SARS-CoV-2 IgY antibodies could be used in nasal or oral spray formulation, we assessed the persistence time of anti-SARSCoV-2 IgY in the nasal and oral cavity of mice by using an in vivo imaging system. To accomplish this, anti-SARSCoV-2 IgY antibodies labeled with fluorescent molecules were administered to $\mathrm{BABL} / \mathrm{c}$ mice via nasal drip or oral spray. At $0,1,2,4,6,8,12$, and $24 \mathrm{~h}$ after administration, respectively, images were captured with the IVIS Imaging System. As shown in Fig. 1I, 1J, anti-SARS-CoV-2-IgY antibodies could persist at a detectable level in the nasal and oral cavities for 2-4 $\mathrm{h}$ and $12-24 \mathrm{~h}$ after administration, respectively. These results suggest that anti-SARSCoV-2 IgY can stay in the upper airways for a matter of hours, depending on the administration method used.

In conclusion, the laboratory-cultured and formaldehyde-inactivated SARS-CoV-2 can be used to immunize hens for large-scale production of egg yolk IgY antibodies with potent inhibitory activity against live and pseudotyped SARS-CoV-2 infection in vitro, as well as SARS-CoV-2 $\mathrm{S}$-mediated cell-cell fusion. In vivo imaging assay showed that anti-SARS-CoV-2 IgY antibodies administered via oral spray or nasal drip could stay in the upper airways for hours. Considering that IgY antibodies are safe for human use and can be manufactured on a large scale with low production cost, these anti-SARS-CoV-2 IgY antibodies have good potential to be further developed as an immunoprophylactic via nasal or oral spray administration to prevent COVID-19. This approach can also be adapted for the development of immunoprophylactics against influenza virus $\mathrm{H} 7 \mathrm{~N} 9$ using the $\mathrm{IgY}$ antibodies from egg yolks of chickens immunized with anti-H7N9 vaccine (Zeng et al. 2018), achieving double effects, i.e., prevention of H7N9 infection in chickens and humans.
Acknowledgements This work was supported by grants from the Key Research and Development Project of Guangdong Province (202020012624900001), the National Natural Science Foundation of China (31830097, 31802174 and 82041025), the Key Research and Development Project of Guangdong Province (2020B1111320001), and the Special fund for the Scientific innovation strategy-construction of high level Academy of Agriculture Science-Prominent Talents (R2020PY-JC001).

\section{Compliance with Ethical Standards}

Conflict of interest The authors declare that they have no conflict of interest.

Animal and Human Rights Statement The mouse experiments were carried out in strict accordance with institutional regulations (approved by the Ethics Committees of the Institute of Process Engineering, Chinese Academy of Sciences, No. 20200610-1).

\section{References}

Abbas AT, El-Kafrawy SA, Sohrab SS, Azhar EIA (2019) IgY antibodies for the immunoprophylaxis and therapy of respiratory infections. Hum Vaccin Immunother 15:264-275

Du L, He Y, Zhou Y, Liu S, Zheng BJ, Jiang S (2009) The spike protein of SARS-CoV-a target for vaccine and therapeutic development. Nat Rev Microbiol 7:226-236

He Y, Li J, Du L, Yan X, Hu G, Zhou Y, Jiang S (2006) Identification and characterization of novel neutralizing epitopes in the receptor-binding domain of SARS-CoV spike protein: revealing the critical antigenic determinants in inactivated SARS-CoV vaccine. Vaccine 24:5498-5508

He Y, Lu H, Siddiqui P, Zhou Y, Jiang S (2005) Receptor-binding domain of severe acute respiratory syndrome coronavirus spike protein contains multiple conformation-dependent epitopes that induce highly potent neutralizing antibodies. J Immunol 174:4908-4915

Hou YJ, Okuda K, Edwards CE, Martinez DR, Asakura T, Dinnon KH 3rd, Kato T, Lee RE, Yount BL, Mascenik TM et al. (2020) SARS-CoV-2 reverse genetics reveals a variable infection gradient in the respiratory tract. Cell 182:429-446.e414

Lee WS, Wheatley AK, Kent SJ, DeKosky BJ (2020) Antibodydependent enhancement and SARS-CoV-2 vaccines and therapies. Nat Microbiol 5:1185-1191

Pereira EPV, van Tilburg MF, Florean E, Guedes MIF (2019) Egg yolk antibodies (IgY) and their applications in human and veterinary health: a review. Int Immunopharmacol 73:293-303

Su S, Du L, Jiang S (2020) Learning from the past: development of safe and effective COVID-19 vaccines. Nat Rev Microbiol. https://doi.org/10.1038/s41579-020-00462-y:1-9

Xia S, Liu M, Wang C, Xu W, Lan Q, Feng S, Qi F, Bao L, Du L, Liu S, Qin C, Sun F, Shi Z, Zhu Y, Jiang S, Lu L (2020) Inhibition of SARS-CoV-2 (previously 2019-nCoV) infection by a highly potent pan-coronavirus fusion inhibitor targeting its spike protein that harbors a high capacity to mediate membrane fusion. Cell Res 30:343-355

Zeng X, Tian G, Shi J, Deng G, Li C, Chen H (2018) Vaccination of poultry successfully eliminated human infection with H7N9 virus in China. Sci China Life Sci 61:1465-1473 